\title{
Level of food intake and the passage of markers and nitrogen along the alimentary tract of sheep
}

\author{
By R. A. WELLER, A. F. PILGRIM AND F. V. GRAY \\ With an Appendix by M. N. TAYLOR, R. A. WELLER and C. S. W. REID* \\ Division of Nutritional Biochemistry, CSIRO, Adelaide, South Australia
}

(Received 24 March I97I - Accepted II May r97I)

\begin{abstract}
I. The relative rates of passage of polyethylene glycol (PEG) and lignin through the alimentary tracts of sheep were examined through the ratio of these two markers in the fodder and in the contents of various segments of the gut. A much faster turnover of PEG than of lignin in the stomach compartments suggested that samples from these organs could not be representative of the digesta flowing through them. Average PEG :lignin ratios in the small and large intestines were close to the ratio in the fodder, indicating that sampling from the duodenum in the living animal could possibly yield material representative of that flowing from the stomach to the small intestine. Similar results were obtained for high and low levels of fodder intake.

2. A procedure was developed for automatic sampling from the duodenum for prolonged periods, and the material collected was shown to be representative of digesta flow. Simpler methods of sampling from the duodenum were shown to be liable to relatively large errors.

3. The amount of nitrogen reaching the duodenum in sheep given lucerne hay pellets was found to be equivalent to practically $100 \%$ of the dietary $\mathrm{N}$, whether the intake of fodder was $600 \mathrm{~g} / \mathrm{d}$ or $1500 \mathrm{~g} / \mathrm{d}$.
\end{abstract}

Precise measurement of the amounts of various substances entering the small intestine under a given feeding regimen obviously forms an important part of digestion studies and this is particularly so in regard to nitrogenous compounds in ruminant digestion. Two main approaches have been under development for some time. The first has involved the use of undigested, unabsorbed markers or reference substances in the diet, analyses being made on the digesta collected before or just after they reach the duodenum. The second approach has aimed simply at collecting and analysing representative samples of the daily flow of digesta through the proximal duodenum.

Probably the earliest example of the first approach in reference to the passage of nitrogenous compounds was reported by Gray \& Pilgrim (1956), who used lignin as a marker. Later work, using a combination of soluble and insoluble markers, indicated that the digesta should be considered as consisting of at least two phases, a fluid phase whose movement the soluble marker would follow and a 'solids' phase for which lignin would be an appropriate marker (Weller, Pilgrim \& Gray, I962). It was shown that the two markers left the rumen at quite different rates and it can be inferred that this would be likely to occur in any reservoirs or diverticula such as the other stomach compartments and might even occur in the lumen of the intestine itself. Further, when one component of the digesta is distributed between the two phases a single marker, soluble or insoluble, could not be used to mark the passage of that component. These facts formed the basis of the development by Hogan \& Weston (1967) of a procedure

* Present address : Applied Biochemistry Division, DSIR, Palmerston North, New Zealand. 
using the two types of marker together. Their method was applied to material collected from the pyloric end of the abomasum and involved a separation of the two phases of the digesta before analysis.

The direct approach through sampling the flow in the proximal duodenum by means of a re-entrant cannula was developed by Hogan \& Phillipson (1960), and considerable modifications of the procedure have been reported by Bruce, Goodall, Kay, Phillipson \& Vowles (1966). In the present work duodenal samples representing digesta flow over prolonged periods were collected automatically and used to measure passage of nitrogen through the proximal duodenum in sheep fed on different amounts of lucerne hay pellets.

\section{EXPERIMENTAL}

\section{Part I. Comparison of the passage of soluble and insoluble undigested markers through the alimentary tract}

This experiment was undertaken in an attempt to find a basis for deciding the validity or otherwise of any method claiming to yield a representative sample of digesta passing along the tract and in particular a sample claiming to be representative of material entering the duodenum in sheep receiving a given diet. The trials were made on ten sheep (Merino wethers) given a daily ration of lucerne hay pellets in equal lots at $\mathrm{I} h$ intervals. Each animal was given the ration for at least 3 weeks, and during the last week the pellets in the diet had been prepared with polyethylene glycol (PEG) present at a concentration of about $10 \mathrm{~g} / \mathrm{kg}$. At the end of the period the sheep were killed, various gut segments were isolated and their contents removed separately for analysis. The ratio PEG:lignin was determined in the fodder and in each of the samples from the tract. Six of the sheep consumed a daily ration of $800 \mathrm{~g}$ and the remainder one of $r 800 \mathrm{~g}$ so that tests were made for two widely different levels of intake.

\section{Part 2. Development and testing of a procedure for sampling the flow of digesta into the proximal duodenum}

The purpose of this part of the work was to develop a procedure yielding samples which could be proved to be representative of digesta flowing to the duodenum over a considerable period (days or weeks if necessary) in order that the average composition could be related to that of the diet and so give reliable information on the extent and nature of digestion in the stomach compartments. Such proof, which has not been available in the procedures previously referred to, was based on the sample having the same ratio of soluble to insoluble marker as that present in the diet and faeces.

Several types of sampling procedure were tested:

(I) Intermittent manual collection of digesta using a simple T-type cannula inserted into the proximal duodenum.

(2) Intermittent manual collection of flow diverted from a re-entrant duodenal cannula.

(3) Automatic sampling based on a mechanical arrangement to divert flow from the re-entrant cannula at fixed intervals over an extended period. 
(4) Automatic removal of a large number of small samples from the cannula, not by allowing digesta to flow from it, but by drawing off material from a small segment of its length without appreciably altering the rate of flow.

(5) Automatic collection over prolonged periods from a stirred compartment incorporated in the re-entrant cannula, arrangements being made for minimum interference with or constraint of the animal.

The apparatus used in types $\mathrm{I}-3$ needs no further description and that used in type 4 was similar in principle to the final apparatus developed in type 5 but did not incorporate a stirring chamber. The final development is described in detail on p. 494 .

Markers. Lignin was the insoluble marker used throughout, both PEG and the complex of chromium with ethylenediaminetetracetic acid (CrEDTA) were used as soluble markers, and they were either incorporated in the pellets or infused continuously into the rumen via a rumen fistula.

Collection and handling of samples. Samples were passed directly to a refrigerator throughout the collection period. Some samples were analysed after short periods of collection, others were allowed to accumulate for $24 \mathrm{~h}$ or more and, when required, portions were taken to make up composite samples representing various periods.

Sheep and feeding. Results for eight sheep (Merino ewes) are reported. They were given equal parts of their daily rations of lucerne hay pellets at $\mathrm{I} h$ intervals. The effects of different levels of intake were examined. The pellets contained $3.05 \% \mathrm{~N}$ and $6.94 \%$ lignin and were prepared from lucerne hay hammer-milled to pass through a sieve with holes of $\frac{3}{16}$ in. diameter.

\section{Part 3. The passage of $N$ to the duodenum in sheep given lucerne hay pellets at low and high levels of intake}

The final experiments from part 2 were used to provide material from which to determine the amounts of $\mathrm{N}$ reaching the duodenum, the ratio of markers being used to check the validity of sampling. The ratio of either marker to $\mathrm{N}$ in comparison with the corresponding ratio in the fodder was determined and the amount of $\mathrm{N}$ reaching the duodenum was then calculated as a proportion of the $\mathrm{N}$ supplied in the diet.

\section{Methods of analysis}

Lignin was determined by the method of Van Soest ( 1963$)$ and $\mathrm{N}$ by the Kjeldahl method. PEG was measured by the method of Hydén (1956) and chromium by atomic absorption. For the estimation of $\mathrm{Cr}$ in duodenal contents $2 \mathrm{ml} \mathrm{II} \mathrm{M-HCl}$ were added per roog sample and the mixture was heated at $100^{\circ}$ for $\mathrm{I}$ h. Recovery tests showed that omission of this step could lead to $\mathrm{Cr}$ values being up to $10 \%$ low in some duodenal samples.

\section{RESULTS}

\section{Part $\mathrm{r}$. Passage of markers along the alimentary tract}

A representative sample of digesta in a particular part of the tract may or may not have the same composition as that leaving the place of sampling. This part of the work was done before part 2, as a preliminary test of the likelihood of our being able to 
obtain from any particular part of the tract a true sample of the digesta flowing through that part.

Results for the ten sheep, summarized in Table $\mathrm{I}$, give a clear picture of the relative rates of passage of the two markers and therefore of the solid and fluid phases of the digesta. Turnover of PEG can be seen to have been much faster than that of lignin in the stomach compartments at both high and low levels of intake. In the upper small intestine the ratio PEG:lignin, expressed as a percentage of the ratio in the diet was very variable $(73-155 \%)$. Similar variation occurred in the lower part of the small

Table I. Ratio PEG:lignin in gut segments, expressed as a percentage of the ratio polyethylene glycol:lignin in the diet (lucerne hay pellets)

\begin{tabular}{cccccccrrr}
$\begin{array}{c}\text { Sheep } \\
\text { no. }\end{array}$ & $\begin{array}{c}\text { Intake } \\
\text { of hay } \\
\text { (g/d) }\end{array}$ & \multicolumn{2}{c}{ Rumen } & Omasum & $\begin{array}{c}\text { Abo- } \\
\text { masum }\end{array}$ & $\overbrace{\text { Upper }}^{\text {Small intestine }}$ & Lower & $\begin{array}{r}\text { Caecum } \\
\text { and } \\
\text { colon }\end{array}$ & Rectum \\
I & 800 & 42 & 28 & 48 & 104 & 105 & 100 & 103 \\
2 & 800 & 37 & 24 & 31 & 105 & 76 & 93 & 100 \\
3 & 800 & 34 & 19 & 33 & 121 & 107 & 116 & 100 \\
4 & 800 & 35 & 15 & 27 & 98 & 100 & 110 & 98 \\
5 & 800 & 19 & 13 & 18 & 73 & 64 & 99 & 104 \\
6 & 800 & 33 & 15 & 37 & 110 & 96 & 94 & 106 \\
Mean & & 33 & 19 & 32 & 102 & 91 & 102 & 102 \\
7 & 1800 & 34 & 22 & 37 & 117 & 104 & 126 & 109 \\
8 & 1800 & 28 & 16 & 29 & 155 & 76 & 99 & 104 \\
9 & 1800 & 26 & 38 & 52 & 85 & 86 & 97 & 94 \\
10 & 1800 & 29 & 20 & 35 & 107 & 107 & 115 & 113 \\
Mean & & 29 & 24 & 38 & 116 & 93 & 109 & 105 \\
General & & 32 & 21 & 35 & 108 & 92 & 105 & 103 \\
mean & & & & & & & &
\end{tabular}

intestine. These values were each for a particular moment and could indicate a fairly irregular passage of the two phases of the digesta over any short interval of time. The mean values for the ten sheep, however, must have been more significant in relation to the average composition of digesta over a longer period for a given diet. The mean of $108 \%$ for the upper small intestine suggests that the markers in this region were likely to be present in proportions similar to those in the diet, provided the mean covered a sufficient length of time. In other words, the average composition of the duodenal contents, unlike the abomasal contents, could be expected to be that of material passing from the abomasum to the small intestine.

The individual values for the large intestine were also variable, but again the means for both high and low intake trials were close to $100 \%$ and the same was so for faeces collected from the rectum.

\section{Part 2. Procedures for sampling from the duodenum}

The simpler methods under (I) to (3) did not encourage us to think that the samples would be satisfactory. In all the tests the ratio of fluid marker to solids marker was significantly lower than the ratio of the markers ingested or introduced, implying that too small a part of the fluid compared with the solids phase of the digesta was collected. 
Some of the results are summarized in Table 2, where average values for as many as fifteen samples, collected over a period of up to $5 \mathrm{~d}$ almost all show a ratio below $80 \%$ and never above $85 \%$ of the ratio in the diet. Much more promising results were obtained with method (4), since with some sheep, when that method was used, the average ratios of soluble to insoluble marker remained close to $100 \%$ of the ratio in

Table 2. Tests of duodenal samples collected by methods (1)-(3) (see p. 488), involving direct flow from cannula to collecting vessel

\begin{tabular}{|c|c|c|c|c|c|}
\hline \multirow{3}{*}{$\begin{array}{l}\text { Method no. } \\
\text { (I) (manual) }\end{array}$} & \multicolumn{5}{|c|}{ Duodenal sampling } \\
\hline & $\begin{array}{c}\text { Sheep } \\
\text { no. }\end{array}$ & $\begin{array}{l}\text { Intake } \\
\text { of hay } \\
(\mathrm{g} / \mathrm{d})\end{array}$ & Frequency & $\begin{array}{l}\text { Collec- } \\
\text { tion } \\
\text { period } \\
\text { (d) }\end{array}$ & $\begin{array}{l}\text { Ratio of PEG : lignin } \\
\text { in the duodenum as } \% \\
\text { of ratio in the diet }\end{array}$ \\
\hline & $\mathbf{I}$ & 1000 & 3 times $/ \mathrm{d}$ & 5 & 76 \\
\hline & 2 & 1000 & 3 times $/ \mathrm{d}$ & 5 & 54 \\
\hline (2) (manual) & 3 & $I 500$ & 2 times/d & 5 & $7 \mathrm{I}$ \\
\hline & 3 & I 500 & 2 times/d & 5 & 85 \\
\hline & 3 & 500 & 2 times/d & 5 & 60 \\
\hline (3) (automatic) & 3 & 1000 & 4 times $/ \mathrm{h}$ & $\mathbf{I}$ & 84 \\
\hline & 3 & 1000 & 4 times $/ \mathrm{h}$ & I & 77 \\
\hline & 3 & 1000 & 4 times $/ h$ & $\mathbf{I}$ & 79 \\
\hline & 3 & 1000 & 4 times $/ \mathrm{h}$ & I & 76 \\
\hline
\end{tabular}

Table 3. Tests of duodenal samples collected by method (4) (automatic collection and bulking of equal-sized samples from within the cannula)

\begin{tabular}{|c|c|c|c|c|c|c|c|}
\hline \multirow{3}{*}{$\begin{array}{l}\text { Sheep } \\
\text { no. }\end{array}$} & \multirow{3}{*}{$\begin{array}{c}\text { Intake } \\
\text { of hay } \\
\text { (g/d) }\end{array}$} & \multirow[b]{3}{*}{ Procedure } & \multirow{3}{*}{$\begin{array}{l}\text { Samp- } \\
\text { ling } \\
\text { period } \\
\text { (d) }\end{array}$} & \multicolumn{4}{|c|}{$\begin{array}{l}\text { Ratio of markers in duodenum as } \\
\% \text { of ratio in the diet }\end{array}$} \\
\hline & & & & \multicolumn{2}{|c|}{ PEG :lignin } & \multicolumn{2}{|c|}{ Cr:lignin } \\
\hline & & & & Range & Mean & Range & Mean \\
\hline \multirow[t]{2}{*}{3} & \multirow{2}{*}{$\begin{array}{r}1000 \\
800\end{array}$} & \multirow{2}{*}{$\begin{array}{l}\text { Collections bulked over } \\
\text { I d for analysis }\end{array}$} & 8 & $93^{-1} 32$ & 102 & - & 一 \\
\hline & & & I 5 & $88-145$ & II 2 & $82-I 3 I$ & 99 \\
\hline \multirow[t]{2}{*}{5} & \multirow{2}{*}{$\begin{array}{r}1500 \\
600\end{array}$} & \multirow{2}{*}{$\begin{array}{l}\text { Collections bulked over } \\
7 \mathrm{~d} \text { for analysis }\end{array}$} & $2 \mathrm{I}$ & $84-95$ & 89 & - & - \\
\hline & & & 28 & $75-81$ & 79 & $7 I-8 I$ & 75 \\
\hline 5 & I 500 & $\begin{array}{l}\text { Collections bulked over } \\
7 \mathrm{~d} \text { for analysis }\end{array}$ & $\begin{array}{l}35 \\
28\end{array}$ & $\frac{-}{110-121}$ & - & $\begin{array}{r}75-\text { I I } 5 \\
104-I 28\end{array}$ & $\begin{array}{r}95 \\
115\end{array}$ \\
\hline
\end{tabular}

PEG, polyethylene glycol.

the diet over periods of $5 \mathrm{~d}$ or more. Nevertheless, as can be seen in Table 3, one of the two sheep tested gave unsatisfactory results. Close observation of the apparatus in action showed that separation of the digesta into fairly distinct layers of more fluid and more solid material often took place within the duodenal cannula. The extent of this separation varied with different animals and different levels of feeding. It was therefore decided to aim at a more satisfactory procedure by maintaining continuous stirring of the digesta flowing through the re-entrant cannula. Results obtained with the apparatus finally evolved and given in Table 4 indicate a significant improvement 
in the accuracy of sampling when the stirring technique was introduced. Comparing the general mean results for sixteen $7 \mathrm{~d}$ collections from each of the two types of sampler, the ratio $\mathrm{Cr}$ :lignin, expressed as a percentage of the ratio in the diet, was 94 (SD I8) with method (4), whereas with the apparatus finally evolved it was 99 (SD $3 \cdot 8$ ).

Table 4. Tests of duodenal samples collected by method (5) (automatic sampling from a stirred reservoir within the cannula)

$\begin{array}{ccccc}\begin{array}{c}\text { Sheep } \\ \text { no. }\end{array} & \begin{array}{c}\text { Intake } \\ \text { of hay } \\ (\mathrm{g} / \mathrm{d})\end{array} & \begin{array}{c}\text { No. of sampling periods } \\ \text { (samples for } 7 \mathrm{~d} \\ \text { bulked for analysis) }\end{array} & \begin{array}{c}\text { Range } \\ \% \text { of ratio in the diet }\end{array} & \text { Mean } \\ 3 & 600 & 2 & 94-106 & 100 \\ 6 & 600 & 4 & 94-98 & 96 \\ 7 & 600 & 4 & 96-103 & 99 \\ 8 & 1500 & 4 & 96-103 & 101 \\ 100-103 & 102\end{array}$

Table 5. Passage of nitrogen to the duodenum in sheep given lucerne hay pellets

\begin{tabular}{|c|c|c|c|c|c|c|}
\hline \multirow{3}{*}{$\begin{array}{c}\text { Sheep } \\
\text { no. }\end{array}$} & \multirow{3}{*}{$\begin{array}{c}\text { Intake } \\
\text { of hay } \\
(\mathrm{g} / \mathrm{d})\end{array}$} & \multirow{3}{*}{$\begin{array}{c}\text { No. of } \\
\text { consecutive } \\
7 \mathrm{~d} \\
\text { samples }\end{array}$} & \multicolumn{4}{|c|}{$\mathrm{N}$ reaching the duodenum as $\%$ of dietary $\mathrm{N}$} \\
\hline & & & \multicolumn{2}{|c|}{ Calculated on lignin basis } & \multicolumn{2}{|c|}{ Calculated on $\mathrm{Cr}$ basis } \\
\hline & & & Range & Mean & Range & Mean \\
\hline 3 & 600 & 2 & $92-106$ & 99 & $99-100$ & 100 \\
\hline 6 & 600 & 4 & $99-107$ & 102 & $102-112$ & 106 \\
\hline 7 & 600 & 4 & $84-98$ & 90 & $90-94$ & 92 \\
\hline 7 & I 500 & 4 & $9 \mathrm{I}-\mathrm{IO} 4$ & 96 & $89-100$ & 95 \\
\hline 8 & 1500 & 2 & $88-120$ & 104 & $88-106$ & 97 \\
\hline
\end{tabular}

Part 3. Passage of $N$ to the duodenum

The amounts of $\mathrm{N}$ reaching the duodenum, expressed as percentages of the amounts of $\mathrm{N}$ given in the diet, were calculated on the basis of both solid and liquid markers and the results are shown in Table 5. As expected from the sampling tests, the values for the two markers agreed closely. At both low and high levels of intake of lucerne hay pellets the amount of $\mathrm{N}$ reaching the proximal duodenum was nearly equal to the amount eaten.

\section{DISCUSSION}

It has been stated that two basically different approaches have been made in the study of the amount of $\mathrm{N}$ reaching the duodenum, but whichever is employed it is necessary that a truly representative sample of the digesta approaching or flowing through the intestine must be obtained. It is possible that a sample drawn at one particular moment from the duodenum could be representative of material leaving the abomasum at that time, but it is at least unlikely that its composition would always be indicative of the average extent of digestion in the stomach. In other words, as suggested by Bruce et al. (1966), the composition of the digesta is likely to be variable. It is clear that one must know the variation of such samples with time and in relation 
to the manner of feeding in order to draw correct conclusions about digestion of the diet in the stomach or the passage of digesta to the intestine. Thus there are two criteria to be met: $(a)$ individual samples must be shown to be representative of the whole contents at the site of sampling at the time they are taken, and $(b)$ a sample must be taken over a sufficiently long period to ensure that its composition corresponds to the average composition of material entering the intestine as the result of the first stages of digestion of the diet in the stomach - that is, that the material is characteristic for the diet in the animal examined.

For our purposes, where the diet contains both soluble and insoluble markers, the criteria can be stated as follows:

(r) Any collection of digesta from the proximal duodenum must have these markers present in the same ratio as in the fodder and faeces if its analysis is to be used to indicate accurately the extent of digestion of dietary components by the time they leave the abomasum.

(2) Since the extent of digestion may vary from time to time during a day or between days, it is necessary to make collections over a sufficiently long period to show that steady average values for digestion are obtained. The results will then be characteristic of the diet, the manner of feeding and the animal.

The first of these criteria has already been used by Hume, Moir \& Somers (1970) in testing the validity of samples taken through an omasal fistula. The authors were satisfied with an insoluble:soluble marker ratio which was $90 \%$ (coefficient of variation $21.6 \%$ ) of the ratio in the diet, and pointed out that values from the work of Hogan (1964), sampling from a re-entrant duodenal cannula, gave similar results $(88 \%$, coefficient of variation $20.3 \%)$. Hogan explained his results as being due to some digestion of the insoluble marker (lignin) between pylorus and anus, but in the light of present work a more likely explanation for both findings may be that the samples were not entirely representative of the digesta flowing from the rumen or abomasum.

A somewhat similar effect is seen in the work of Bruce et al. (1966), who could not recover the theoretical amount of added $\mathrm{Cr}_{2} \mathrm{O}_{3}$ in their samples from a simple reentrant duodenal cannula when the whole of the passing digesta were collected and sampled. In that work too the sample was probably not characteristic of the normal digesta but the reason is more likely to be found in the necessarily more extensive interference with normal flow through the tract. It is not possible to say precisely how effective the authors' corrections, based on $\mathrm{Cr}_{2} \mathrm{O}_{3}$ recovery, would be in calculating true flow-rates, or what effect incorrect sampling might have on the composition attributed to the digesta.

One of the aims of the present work on sampling procedure was to cause a minimum disturbance of the normal flow of digesta from the abomasum, and as little handling of the animal as practicable, by making the procedure automatic. In the procedure, sampling at 5 or $10 \mathrm{~min}$ intervals has continued for as long as 6 weeks without any apparent undesired effects on the sheep. Tests of the sampling method were limited to the use of regular hourly feeding of ground material, but it is likely that, with sufficiently prolonged periods of continuous sampling and because of the limited size 
of duodenal digesta particles, the procedure should be applicable to irregularly fed sheep eating unground hay. Further trials are planned to include these factors.

We are indebted to Dr C. S. W. Reid and Dr G. H. McIntosh for the surgical preparations. We also thank Dr Reid for his interest and suggestions during the development of duodenal sampling procedures.

\section{APPENDIX}

\section{A method for automatic sampling of digesta from the duodenum}

Automatic sampling of material flowing through the duodenum of cannulated caged sheep has been achieved with the possibility of prolonged periods of continuous collection and with little disturbance to the animal. The external section of a reentrant duodenal cannula includes a reservoir within which digesta are continuously stirred and from which samples are withdrawn automatically at regular intervals.

A diagram of the equipment is shown in Fig. I (a). The reservoir A of about $70 \mathrm{ml}$ capacity is inserted into the cannula and its contents are stirred by a helical rotor driven by a geared motor at $500 \mathrm{rev} / \mathrm{min}$ through a flexible drive $B, 2.5 \mathrm{~m}$ long. The sampling unit $A^{\prime}$ is operated through a Bowden cable $E$ at regular intervals, and during each operation it delivers about ${ }^{1} \cdot 5 \mathrm{~g}$ duodenal contents through an outlet tube $D$. Expulsion is aided by momentary gas pressure through the tube $G$ and the sample passes to a refrigerated storage bottle. Triple support for the sampler is provided by the cannula, by a counterweight $C$ and by tapes attached to sheet-rubber cemented to the animal's back. The control unit $F$ contains a motor geared to $3 \mathrm{rev} / \mathrm{min}$ with switching so arranged that it makes a single revolution following an impulse from a timer. A crank on the motor shaft operates $E$, controlling the position of a plunger within $A^{\prime}$. On the same shaft is a cam which operates a valve allowing a small pressure of nitrogen in $G$ at the expulsion stage of the sampling cycle. All connexions $(B-E, G)$ are sufficiently flexible to allow freedom of movement of the animal within its cage.

Sampler construction. Details of the sampling unit are shown in Fig. $\mathrm{I}(b, c)$. The casings of the reservoir and sampler are turned from brass to a wall thickness of I $\mathrm{mm}$ and silver soldered together. Glass linings are provided by appropriately cut portions of a $100 \mathrm{ml}$ beaker and a $25 \mathrm{ml}$ syringe barrel, these being sealed in position with silicone rubber cement. Sections of the brass casings are cut away to leave glass windows in both the reservoir and the sampler. Brass nipples are soldered to the sampler for attachment of gas inlet and sample outlet tubes. The internal diameter of the outlet nipple should be not less than $7 \mathrm{~mm}$. The duodenal flow inlet tube $N$ is cut longitudinally, spread and soldered to the wall of the stirring chamber to give a minimum of projection without loss of cross-sectional area. A baffle $O$ is fixed above $N$ to deflect flow into the reservoir. Silicone putty is moulded $(Y)$ to streamline the stirring chamber.

The top of the chamber is sealed by a neoprene gasket and a lid, $L$, attached by four screws. The lid carries the duodenal flow outlet $N^{\prime}$ and the rotor assembly. A sealed ball-bearing unit $H$ is fixed to the Bowden cable coupling, $B^{\prime}$, and to $L$ through a 
support (not shown in the diagram). Where the spindle, $\mathcal{F}$, passes through $L$ a seal is completed by the adjustable felt gland $K$. The hollow shaft of the rotor, $M$, is slit and sprung to provide a close fit on $\mathcal{f}$ and a clutch mechanism should $M$ become jammed.
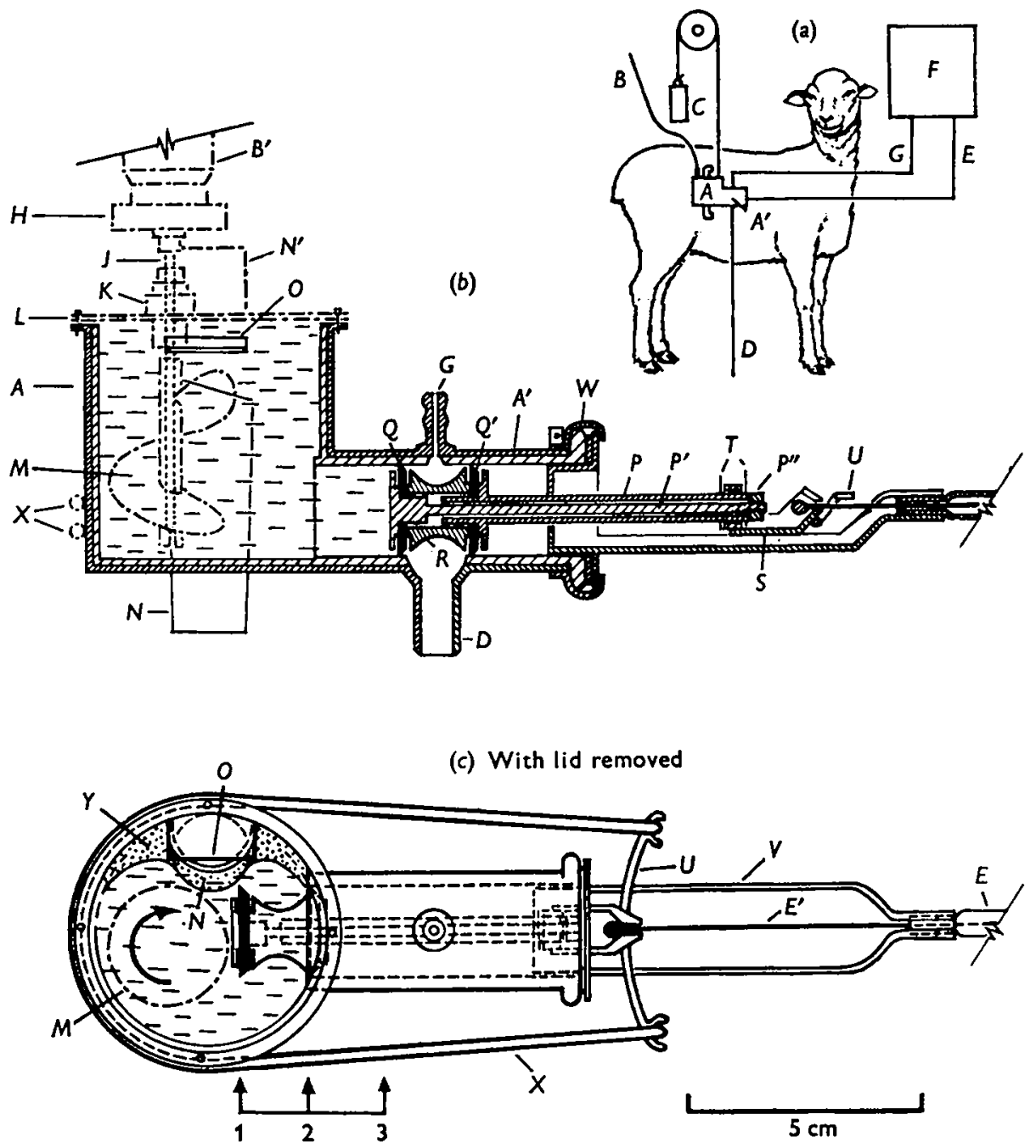

Fig. x. Apparatus for automatic sampling of digesta from the duodenum.

(a) Diagrammatic representation of attachments to the sheep. $A$, stirred reservoir in reentrant cannula; $A^{\prime}$ sampling syringe; $B$, flexible drive for stirrer; $C$, counterweight; $D$, sample outlet; $E$, Bowden cable; $F$, sampling control unit; $G$, gas pressure tube.

(b) Elevation section with plunger in expulsion position.

(c) Plan section with plunger in sampling position.

Details of components $A-Y$ are given on pp. $494-496$.

The shaft of the sampling plunger is formed from a tube $P$ through which passes a threaded $\operatorname{rod} P^{\prime}$. Both $P$ and $P^{\prime}$ are flanged at one end and a capstan nut $P^{\prime \prime}$ is used to adjust the distance between the flanges. A sliding seal to the inside of the syringe 
barrel is provided by two resilient washers, $Q, Q$ ', moulded from 'Corvic' paste (ICI, P 65-50). Between the washers is a reel-shaped nylon spacer $R$ which determines the sample size. The surfaces of $P, P^{\prime}$ and $R$ adjacent to the washers are stepped as shown, so that when $P^{\prime \prime}$ is tightened compression of the washers is near the centre while the outer edges remain loosely held. Final adjustment of $P^{\prime \prime}$ is made under operating conditions.

The inner core, $E^{\prime}$, of the Bowden cable is attached to the plunger through a linkage, $S$, which fits over $P$ and is held in position by two circlips, $T$. Attached to $S$ is a yoke, $U$, with hooks to hold the stretched rubber band $X$. The Bowden cable casing is held by a support, $V$, attached to the sampler body by a clamp, $W$.

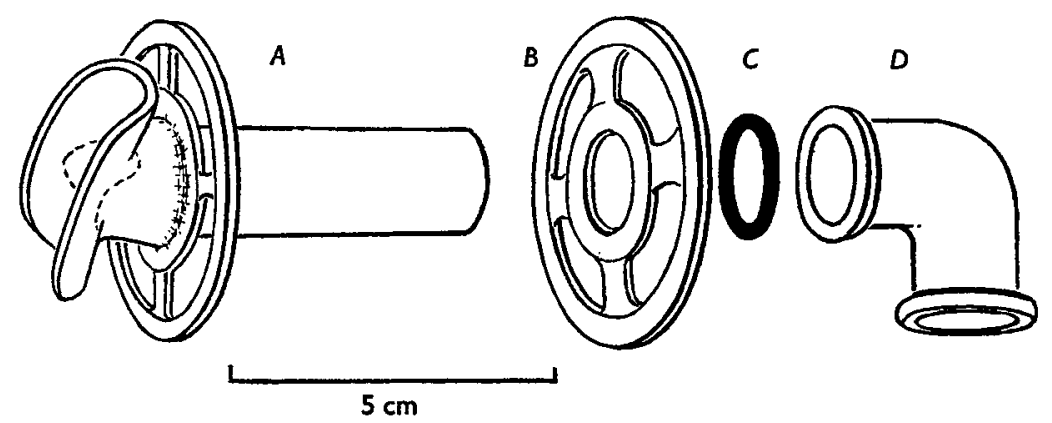

Fig. 2. Duodenal cannula. $A$, stem and inner flange moulded from polypropylene in one piece; $B$, outer flange; $C$, rubber retaining ring; $D$, elbow moulded from 'Corvic' PVC paste (ICI, P65-50).

Sampler operation. The rotor, $M$, turns with a downward thrust and maintains a uniform suspension of duodenal material, while the action of the plunger is intermittent. The plunger positions, with $Q$ as a reference, are shown below Fig. I $(c)$. During the operating cycle the plunger moves from the resting position (2) to the sampling position (I) then to expulsion position (3) and finally back to 2 . The duration of this cycle is $20 \mathrm{~s}$. The volume of bulked sample collected may be adjusted to a suitable proportion of the total duodenal flow by selecting an appropriate interval between samples. For example, in the experiments reported above intervals of 5 or Io min, giving samples of about $400 \mathrm{~g}$ or $200 \mathrm{~g} / \mathrm{d}$, were used with high and low levels of feeding respectively. With both sizes of sample this represented about $3 \%$ of the daily flow through the duodenum as measured by marker dilution.

Re-entrant cannula. Surgical preparation of the animal involved transection of the duodenum about $6 \mathrm{~cm}$ from the pylorus and the fitting of a polypropylene cannula, with an internal diameter of $12 \mathrm{~mm}$, of a design shown in Fig. 2. The stems were brought through the body wall in such positions that with the animal standing the distal cannula was about $10 \mathrm{~cm}$ vertically above the proximal. Flexible PVC elbows attached to the stems allowed connexion of the cannulas through a length of perspex tube or through the sampling apparatus. 
Vol. 26

\section{REFERENCES}

Bruce, J., Goodall, E. D., Kay, R. N. B., Phillipson, A. T. \& Vowles, L. E. (1966). Proc. R. Soc. B $166,46$.

Gray, F. V. \& Pilgrim, A. F. (1956). Nature, Lond. 178, 94.

Hogan, J. P. (1 964). Aust. F. agric. Res. I5, 384 .

Hogan, J. P. \& Phillipson, A. T. (1960). Br. Y. Nutr. I4, I47.

Hogan, J. P. \& Weston, R. H. (1967). Aust. F. agric. Res. 18, 803 .

Hume, I. D., Moir, R. J. \& Somers, M. (I970). Aust. $\mathcal{~}$. agric. Res. 21, 283.

Hydén, S. (1956). LantbrHögsk. Annlr 22, 139.

Van Soest, P. J. (1963). F. Ass. off. agric. Chem. 46, 829.

Weller, R. A., Pilgrim, A. F. \& Gray, F. V. (1962). Br. J. Nutr. I6, 83. 